\title{
Mini Review: The Mental and Physical Consequences of Chinese Shidu Parents
}

\author{
Qianlan Yin*, Zhuoer Sun\#, Weizhi Liu* \\ Faculty of Psychology and Mental Health, Second Military Medical University, Shanghai, China
}

Article Info

\section{Article Notes}

Received: March 23, 2018

Accepted: May 10, 2018

\section{${ }^{*}$ Correspondence:}

Dr. Weizhi Liu, Ph.D, MD, Faculty of Psychology and Mental Health, Second Military Medical University, Shanghai, China;

Email: liuweizhi@smmu.edu.cn;

"Equal contributors

(c) 2018 Liu W. This article is distributed under the terms of the Creative Commons Attribution 4.0 International License.

\begin{abstract}
"Shidu Parents" (SDP) means parents whose only child has passed away or is disabled to perform the basic social function and daily living activities due to an accident or other events. These parents cannot conceive or adopt another child. Bereavement is a great grief and threat to mental and physical states evidenced by many studies, but SDP are a new and increasing subgroup emerging in this field, especially in China. The goal of this paper is to review the research literature regarding mental and physical health consequences of SDP and the intertwined influence, thus to add to the corresponding assistance from the government.
\end{abstract}

\section{Introduction}

"Shidu Parents" (SDP) means parents whose only child has passed away or is disabled to perform the basic social function and daily living activities (officially above level 3 disabled) due to an accident or other events. These parents cannot conceive or adopt another child ${ }^{1}$. These parents may be also referring to Parents of losing the only child (PLOC) or Shiduers in some studies but the SDP is more official. The number of one-child families in China has increased dramatically in the past 30 years as a result of the only-child policy ${ }^{2-4}$. Based on the population census statistics in 2011, the total number of Shidu family reached four hundred and forty-seven thousand in the nation with seven thousand and six hundred Shidu families occurring each year ${ }^{1,5}$. (Source: http://news. xinhuanet.com/local/2013-03/02/c_124406508.htm, last accessed on 29 March 2016). Thus, given the challenges of being SDP for the increasing population, the aim of this paper is to analyse the characters of Chinese SDP and the consequences of being SDP which needs to be addressed in order for the government to better assist them.

\section{The difference between SDP and other bereavement}

Bereavement is a severe life event that leads to increased mortality in widowed populations. The death of a child is especially stressful $^{6}$, resulting in a significantly high intensity of unresolved grief, which can worsen mental and physical health ${ }^{7-9}$. Another study also suggested that strong continuing bonds predicted greater levels of traumatic and especially separation distress ${ }^{10}$. Many western studies on parental bereavement have provided significant insights and theoretical perspectives to understand SDP, such as grief theory and sense-making explanation ${ }^{11-14}$, but very 
few of them are specifically about losing an only child, which may raise different concerns from losing other family members in non-western countries. Besides, a lot of researches about other bereavement are conducted to find the disparities in the causes of parental bereavement such as cancer, accidents or suicide ${ }^{15,16}$, instead of the factors of the relationship between children and parents or the amount of the losing children. Based on the 2011 baseline data from the China Health and Retirement Longitudinal Study $^{17}$ whose data cover 17,705 individuals aged 45 or older, living in 10,287 households in 450 rural villages or urban communities with 6,739 observations with complete information, the effects were significantly stronger for losing all the children, similar to losing the only child, than those having a living child or not losing, compared the mental health, happiness and loneliness after adjusting for demographic and socioeconomic characteristics. Previous research also has shown that bereaved parents exhibit more symptoms of depression, anxiety, loneliness, grief, and posttraumatic stress disorder than did their non-bereaved counterparts ${ }^{18,19}$. Meanwhile, bereavement was associated with long-term mortality due to illness (e.g., cancer), presumably because of stress, a weakened immune system, or poor health behaviors (e.g., smoking, alcohol consumption). Therefore, it is supposed that the physical and mental health consequences among SDP could be even worse and more focus on the them should be paid and more studies on the intertwined influence between the mental and physical consequences of SDP.

\section{Dilemma of SDP in China}

Chinese culture is family-oriented and the relationships between parents and children are much closer and very important. Although China is becoming more westernized, Confucianism still represent the core values of Chinese society. One of the values is making the passing on the family name an obligation, failing to do so triggers a great deal of distress ${ }^{20}$. In the Chinese systematically concept, having children is essential for a whole family. The belief is that "having no posterity is extremely non-filial"; those who do not have a child are culturally stigmatized as "juehu"(绝户), which literally means those who are going to become extinct, simply because they failed to take the responsibility of passing on the family name of their ancestors. Chinese culture categorizes the death of an adult child as a "bad" death because the death of children places the parents in a very painful situation literally translated as "the white headed witnesses the death of the black headed" 20 and the culture views the death of children as a result of karma. Besides, research indicated that parents in one-child families demonstrate "child-centeredness" significantly more than parents in multiple-child families ${ }^{21}$. Losing the only child often means losing their main source of care-giving, hope for the future, a great threat to the individual's well-being, and consequences of declining mental health or social withdrawal, which may progress to a point of social isolation because communicating about their child's death is often difficult and can be hurtful and stigmatizing ${ }^{22}$. The dominant Confucian philosophy regard death as a negative event in life ${ }^{23}$; it makes death a taboo topic which hinders acceptance of grief counseling or other forms of professional intervention. In addition, Chinese people pay more value on the blood relationship, and put the family value at the priority, so they are less willing to adopt a child considering their other family members' disagreement.

The implementation of the one-child policy beginning in the late 1970s has further aggravated the negative implications of the death of a child for Chinese families. However, the policy has changed a lot and allows each couple to have two births, but in 2010 there were 145 million people from the only-child family and under the current population policy allowing to have two births, Chinese average fertility rate is only 1.6 (according to the data from the World Bank ) and even in 2050 it will up to 1.8 ( according to the predictable data from the United Nation). The Chinese government has taken measures to mitigate the policy's negative effects. However, these measures are mostly focused on material supports, including increasing the national standard subsidy for parents of the lost only child but studies showed that the increasing subsidy and other financial aids did not improve the situation of SDP where they were deemed as a social identity culturally stigmatized and politically victimized $^{22}$. Some of Shidu parents rejected to receive the financial aids as they didn't want to be pitied for the lose of their children and felt stigmatized for the discontinuity of their family. They may have isolated themselves from the society and concealed or avoided their trauma. In another word, the financial and material aids can arouse their helplessness for living alone in their old age. After all, Shidu is a unique social issue in Chinese society, which deserves a higher level of attention.

\section{Current studies of the Chinese SDP on the mental and physical consequences}

There recently have been some studies investigating a large group of SDP comparing the parents with children, nationally or regionally. These studies showed that losing only child has adverse effects on mental health, loneliness, happiness and general emotional well-being through investigations using a data from the China Health and Retirement Longitudinal Study (CHARLS) 2011 baseline data, which was initiated to study the elderly population of China and published in March 2012 with the measures of a 10-item Center for Epidemiological Studies Depression Scale (CES-D Scale) and other self-adopted questionnaires about variables regarding losing children, Individual demographic, socioeconomic and health status ${ }^{17}$. 
Yongqiang Zheng et al in 2015 compared 42 older adults who lost their only child to 33 older adults who have a child, in term of their physical and mental health, and social support through telephoning, internet questionnaires, face-to-face online interview ${ }^{24}$. The results confirmed the general deteriorating trend and significant impairments in those aspects of SDP using Instrumental Activities of Daily Living scale (IADL), The Geriatric Depression Scale-15 (GDS-15), The Inventory of Complicated Grief (ICG) and The Lubben Social Network Scale (LSNS), comparing to the clinical diagnosis cutoff points used in Western countries. However the specific mental and physical states of SDP have not extensively been evaluated.

A new study in our research team is undertaken to compare SDP (a sample of 95 participant) with their counterparts (97 participants) with a living child by a series of standardized assessments of their physical health and mental health problems such as PTSD and depression ${ }^{25}$. In terms of mental assessments, it adopted the 20-item Center for Epidemiological Studies Depression Scale (CES-D) and PTSD Checklist-Civilian Version (PCL-C). The General Health Questionnaire (GHQ-12) was used to evaluate the severity of general psychiatric morbidity. The results of this study have confirmed SDP have more mental and physical consequences. In detail, $32.6 \%$ of the Shidu group in the study had PTSD, much higher than the percentage or incidence from relevant studies such as Jiong, $\mathrm{L}$ et $\mathrm{al}^{6}$, He, $\mathrm{L}$ et $\mathrm{al}^{26}$ and Shirley et $\mathrm{al}^{27}$ but these studies were all about bereavement of parents not disentangling SDP from them. Furthermore, poor health and symptoms of PTSD were more likely to be observed among the Shidu group even though the Shidu group were wealthier and younger than their counterparts. Besides, women were more likely to develop PTSD and depression than men same as the western studies, which provide some explanation about the role expectations of women compared to $\mathrm{men}^{26}$. Another domestic investigation about mother's grief of losing the only child also provided some valued explanations ${ }^{27}$. Given the role expectation in China, mothers are playing a bigger role in raising their only child, and spend more time with the child than fathers do, so mothers and children have a very closed relationship which may supersede the martial relation, therefore losing the only child raises more challenges for mothers than for fathers. Studies also addressed the physical effects in detail and showed a higher morbidity of coronary heart disease (RatioSDP $=28.4 \%$, Ratiocontrol $=3.1 \%, \mathrm{P}<0.001$ ), tumors (RatioSDP $=7.4 \%$, Ratiocontrol $=1.0 \%, \mathrm{P}=0.028$ ), mental diseases (RatioSDP $=7.4 \%$, Ratiocontrol $=1.0 \%, P=0.028$ ) and other unclassified diseases (RatioSDP $=11.6 \%$, Ratiocontrol $=2.1 \%, \mathrm{P}=0.028$ ) in the Shidu group compared to the control. These diseases were closely related to the long-term psychological suffering of losing the only child. As for the intertwined influences between the mental and physical diseases on SDP, losing the only child diminishes both physical health and mental health of parents, which are closely related and can affect each other in bidirectional relationship.

\section{Improvement and Outlooks}

This review highlights the consequences of losing the only child among Chinese parents referred to SDP in the bereavement literature, which deserves more investigations for better prevention and intervention. There is a relative paucity of rigorous, multi-centered prospective research regarding long-term parent or family outcomes following the death of only child. Indeed, much of the data are descriptive, and/or from retrospective, cross-sectional studies. There are several limitations to this review as we may have not collected all the information on the topic or missed several critical and valuable papers which describe parental perspectives without formal instrumentation. Finally, the challenges that are pervasive in this literature, including small sample sizes and mixed methodologies, make it more difficult to draw strong conclusions.

Taking the current health condition of SDP into consideration, SDP are often unable to effectively perform normal or usual activities of daily living during the postloss time period. They may experience sleep disruption, reduced financial security, less freedom of action, and decreased perceived personal safety, all of which can secondarily affect impaired daily functioning. Therefore, we urge the development of corresponding regulations and services to improve SDP life quality. For instance, we need to enhance health-care to SDP, and to intervene with those who did not go to clinical services regularly or were unwilling to get clinical services. It also should revise some policies such as the elderly people in nursing homes need their children to sign in and the financial support should be flexible and easily accessible. As we interviewed, one participant in our study has been planting thousands of trees in the desert isolated from the society to memorize his son and other SDP may hold their dead child's picture whenever and wherever they are. Therefore, what is more important is that more properly psychological aids should be provided for them. Some western researches about bereavement conclude that interventions that facilitate processes of meaning reconstruction can support effective psychotherapy for those struggling with intense and prolonged grief, which indicate helping SDP to find positive meaning in the experience with resilience and dyadic coping, especially stress communication, can constitute important therapeutic goals ${ }^{28,29,30}$. Further investigations are needed to explore some other predictors responsible for the prevalence of PTSD and depression in SDP, such as the personality of the parents, the characteristics of the lost children, and the social environment, which may contribute a lot to the immediate interventions ${ }^{31}$. 
As a whole, more studies can be beneficial to address the factors causing the differences between the SDP and the parents with a living child, while some case surveys also should be valued as the individual variations are emphasized.

\section{References}

1. Chen, E. The number of the only child died families in China. Population and Development. 2013); 19: 100-103

2. Mu GZ. Aiding and caring the emergency of the family sacrificing for the fertility policy. Population And Development. 2008; 14: 27-30

3. Xiao L,Sun M,Wang QY,et al. The dilemma and copy strategy of the only child died families in China. Chinese Journal of Gerontology. 2016; 36(3): 742-744

4. Guo Z, Zhang E, Gu B, et al.“Diversity of China's Policy by Policy Fertility." Population Research. 2003; 27(5): 1-10

5. The popular research project of Peking University. The study about the caring system of loss-of-single-child families sampled in Liaoning province. Chinese journal of Yanan Cadre Institute. 2011; 4: 50-60

6. Jiong L, Dorthe HP, Preben BM, et al. Mortality in parents after death of a child in Denmark:a nationwide follow-up study.Lancet. 2003; 361: 363-367.

7. Fletcher PN. Experiences in family bereavement. Family and Community Health. 2002; 25: 57-70.

8. Rogers CH, Floyd FJ, Seltzer MM, et al. Long-term effects of the death of a child on parents' adjustment in midlife. Journal of Family Psychology. 2008; 22: 203-211

9. Kristensen P, Weisaeth L, Heir T. Bereavement and mental health after sudden and violent losses: a review. Psychiatry. 2012; 75: 76-97

10. Neimeyer RA, Baldwin SA, Gillies J. Continuing bonds and reconstructing meaning: mitigating complications in bereavement. Death Stud. 2006; 30(8): 715-38

11. Patrizia K. Lannen, Joanne Wolfe, Holly G, et al. Unresolved Grief in a National Sample of Bereaved Parents: Impaired Mental and Physical Health 4 to 9 Years Later. American Society of Clinical Oncology. 2008; 26(36): 5870-5876

12. Titus B, de Souza R. Finding meaning in the loss of a child: journeys of chaos and quest. Health Commun. 2011; 26(5): 450-60.

13. Keesee NJ, Currier JM, Neimeyer RA. Predictors of grief following the death of one's child: the contribution of finding meaning. J Clin Psychol. 2008; 64(10): 1145-63

14. Kim J, Hicks JA. Parental bereavement and the loss of purpose in life as a function of interdependent self-construal. Front Psychol. $2015 \mathrm{Ju}$ 27; 6: 1078 .

15. von Lützau, Pia;Otto, Michael;Hechler, et al.Children Dying from Cancer: Parents' perspectives on symptoms. Journal of Palliative Care. 28:4278.

16. Bolton JM, Au W, Leslie WD, et al. Parents bereaved by offspring suicide: a population-based longitudinal case control study. JAMA Psychiatry. 2013; 70: 158-167

17. Ren QP, Ye ML. Losing children and mental well-being: evidence from China, Applied Economics Letters. 2016. DOI: $10.1080 / 13504851.2016 .1237732$

18. Nyberg T, Hed Myrberg I, Omerov P, et al. Depression among parents two to six years following the loss of a child by suicide: A novel prediction model. Plos One. 2016; 11(10): e0164091.

19. Harper MO, Connor RCO, Carroll RE. Factors associated with grief and depression following the loss of a child: A multivariate analysis. Psychology, Health \& Medicine. 2014; 19(3): 247-52

20. Chan CL, Chow AY, Ho SM, et al. The experience of Chinese bereaved persons: A preliminary study of meaning making and continuing bonds. Death Studies. 2005; 29(10): 923-947

21. Chow ENL, Zhao SM. The one-child policy and parent-child relationships: A comparison of one-child with multiple-child families in China. International Journal of Sociology and Social Policy. 1996; 16(12): 35-62.

22. Zheng Y, Lawson TR. Identity reconstruction as Shiduers:Narratives from Chinese older adults who lost their only child. International Journal of Social Welfare. 2014; 24(4). DOI: 10.1111/ijsw.12139

23. Xu Y. Death and dying in the Chinese culture: Implications for health care practice. Home Health Care Management \& Practice. 2007; 19(5): 412-414

24. Zheng Y, LawsonTR, Barbara AH. "Our Only Child Has Died": A Study of Bereaved Older Chinese Parents. OMEGA-Journal of Death and Dying. 2015; 1 - 16. DOI: 10.1177/0030222815612285

25. Yin QL. An investigation of physical and mental health consequences among Chinese parents who lost their only child. BMC Psychiatry. 2018. DOI 10.1186/s12888-018-1621-2

26. He L, Tang S, Yu W, et al. The prevalence, co-morbidity and risks of prolonged grief disorder among bereaved Chinese adults. Psychiatry Research. 2014; 219(2): 347-352.

27. Shirley A, Murphy L, Clark Johnson, Ick-JoongChung, et al. The Prevalence of PTSD Following the Violent Death of a Child and Predictors of Change 5 Years Later. Journal of Traumatic Stress. 2003; 16: $17-25$

28. Fang F, Lin C, Wei S, et al. Mothers' Grief Experiences of Losing Their Only Child in the 2008 Sichuan Earthquake: A Qualitative Longitudinal Study. Journal of Death and Dying. 2018; 1-15.DOI: $10.1177 / 0030222818755287$

29. Marissa K. Pfoff, Joette R. Zarotney,Timothy H. Monk. Can a functionbased therapy for spousally bereaved senior accrue benefits in both functional and emotional domains. Death Stud. 2014; 38(6): 381-386

30. Sara Albuquerque, Isabel Narciso, Marco Pereira. Posttraumatic Growth in Bereaved Parents: A Multidimensional Model of Associated Factors, Psychological Trauma: Theory, Research, Practice, and Policy. 2018; 10(2): 199-207

31. Lee C, Glei DA, Weinstein M, et al. Death of a child and parental wellbeing in old age: Evidence from Taiwan. Social Science and Medicine. 2014; 101: 166-173 